\title{
Universality classes in directed sandpile models
}

\author{
Romualdo Pastor-Satorras and Alessandro Vespignani \\ The Abdus Salam International Centre for Theoretical Physics (ICTP), P.O. Box 586, 34100 Trieste, Italy
}

(March 29, 2021)

\begin{abstract}
We perform large scale numerical simulations of a directed version of the two-state stochastic sandpile model. Numerical results show that this stochastic model defines a new universality class with respect to the Abelian directed sandpile. The physical origin of the different critical behavior has to be ascribed to the presence of multiple topplings in the stochastic model. These results provide new insights onto the long debated question of universality in abelian and stochastic sandpiles.
\end{abstract}

PACS numbers: 05.65.+b, 05.70.Ln

The class of sandpile models, consisting of the original Bak, Tang and Wiesenfeld (BTW) [1] automata and its theme variations, is considered the prototypical example of a special class of driven non-equilibrium systems exhibiting a behavior dubbed self-organized criticality (SOC). Under an external drive, these systems spontaneously evolve into a stationary state. In the limit of infinitesimal driving the stationary state shows a singular response function associated to an avalanche-like dynamics, indicative of a critical behavior. Sandpile models have thus attracted a great deal of interest, as plausible candidates to explain the avalanche behavior empirically observed in a large number of natural phenomena [2].

In recent years, the possibility of understanding the sandpile critical behavior in analogy with other nonequilibrium critical phenomena such as branching pro-

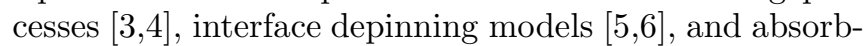
ing phase transitions [7,8] has been pointed out. It is then most important to identify precisely, for sandpiles, the universality classes and upper critical dimensions, which are basic and discriminating features of the critical behavior. Despite significant numerical efforts, however, these issues remain largely unresolved. For instance, it is still an open problem wheter or not the original deterministic BTW sandpile and the stochastic Manna two-state model [9] belong to the same universality class. Theoretical approaches 10 12 support the idea of a single universality class, while numerical simulations provide contradictory results 13315$]$.

In order to have a deeper understanding of the universality classes puzzle, we turn our attention to directed sandpile models [16]. In this case Dhar and Ramaswamy obtained an exact solution for the Abelian directed sandpile (ADS) [16], that can be used as a benchmark to check the numerical simulation analysis. Directed sandpiles thus become an interesting test field to study how critical behavior is affected by the introduction of stochastic elements. Despite the fact that results obtained for directed models cannot be exported "tout court" to the isotropic ones, the eventual apparence of different universality classes provides interesting clues on the general problem of universality in sandpiles. This issue has been recently addressed in a particular case by Tadić and Dhar [17], but a general discussion of universality classes in di- rected sandpile automata is still lacking.

In this letter we present large scale numerical simulations of Abelian and stochastic directed sandpile (SDS) models. First, we study an ADS model for which we recover numerically the results expected from the analytical solution [16]. Then we introduce a stochastic model which is a directed version of the Manna two-state sandpile [9]. In this case, the set of critical exponents defines a different universality class. For both models we provide a very accurate study of finite size effects and the convergence to the asymptotic behavior. For small and medium lattice sizes we find scaling anomalies that are similar to those encountered in isotropic models. We also study in detail the geometrical structure of avalanches. The presence of multiple topplings appears to be the fundamental difference between Abelian and stochastic models. Numerical simulations in Euclidean dimension $d>2$ show that both universality classes have an upper critical dimension $d_{c}=3$, where strong logarithmic corrections to scaling are present.

We consider the following definition for an ADS model (see Fig. 1(a)): On each site of a $d$-dimensional hypercubic lattice of size $L$, we assign an integer variable $z_{i}$, called "energy". Each time step, an energy grain is added to a randomly chosen site $\left(z_{i} \rightarrow z_{i}+1\right)$. When a site acquires an energy greater than or equal to the threshold $z_{c}=2 d-1$, it topples. Topplings are directed along a fixed direction $x_{\|}$(defined usually as "downwards"): when a site on the hyperplane $x_{\|}$topples, it sends deterministically one energy grain to each nearest and nextnearest neighbor site on the hyperplane $x_{\|}+1$, for a total of $2 d-1$ grains. This definition differs from the ADS studied by Dhar and Ramaswamy [16] in the orientation of the lattice. Both models, however, share the same universality class, being Abelian, deterministic, and directed.

The stochastic generalization of the above model is depicted in Fig. If(b): The threshold is now $z_{c}=2$, independent of the spatial dimension. When a site at the hyperplane $x_{\|}$topples, it sends two grains of energy to two sites, randomly chosen among its $2 d-1$ neighbors in the hyperplane $x_{\|}+1$. The dynamical rule of this model can be defined exclusive if the two energy grains are always distributed on different sites. On the contrary, a nonex- 


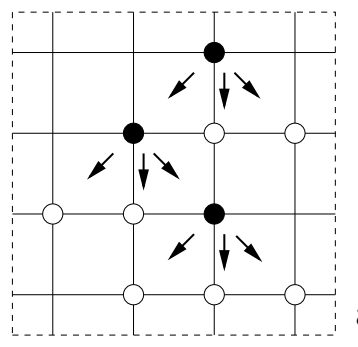

a)

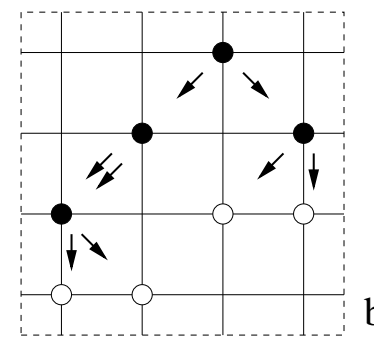

b)

FIG. 1. Toppling rules in $d=2$ for directed sandpiles. Filled circles represent active (toppling) sites; empty circles are stable sites. In the Abelian model (a) an active site sends one grain to each one of its three neighbors on the next downwards row. In the stochastic models (b) one grain is sent to two randomly chosen downwards neighbors.

clusive dynamics allows the transfer of two energy grains to the same site. We will consider separately the cases of the exclusive stochastic directed sandpile (ESDS) and the nonexclusive stochastic directed sandpile (NESDS). It is worth remarking that stochasticity does not alter the Abelian nature of the model [18]. All three models are locally conservative; no energy grains are lost during a toppling event. Boundary conditions are periodic in the transverse directions and open at the bottom hyperplane $x_{\|}=L$, from which energy can leave the system.

In the critical stationary state, we can define the probability that the addition of a single grain is followed by an avalanche of toppling events. Avalanches are then characterized by the number of topplings $s$, and the duration $t$. According to the standard finite size scaling (FSS) hypotesis, the probability distributions of these quantities are described by the scaling functions

$$
\begin{gathered}
P(s)=s^{-\tau_{s}} \mathcal{G}\left(s / s_{c}\right), \\
P(t)=t^{-\tau_{t}} \mathcal{F}\left(t / t_{c}\right),
\end{gathered}
$$

where $s_{c}$ and $t_{c}$ are the cutoff characteristic size and time, respectively. In the critical state the lattice size $L$ is the only characteristic length present in the system. Approaching the thermodynamic limit $(L \rightarrow \infty)$, the characteristic avalanche size and time diverge as $s_{c} \sim L^{D}$ and $t_{c} \sim L^{z}$, respectively. The exponent $D$ defines the fractal dimension of the avalanche cluster and $z$ is the usual dynamic critical exponent. The directed nature of the model introduces a drastic simplification, since it imposes $z=1$. A general result concerns the average avalanche size $\langle s\rangle$, that also scales linearly with $L$ [16, 19, 20, : a new injected grain of energy has to travel, on average, a distance of order $L$ before reaching the boundary. In the stationary state, to each energy grain input must correspond, on average, an energy grain flowing out of the system. This implies that the average avalanche size corresponds to the number of topplings needed for a grain to reach the boundary; i.e. $\langle s\rangle \sim L$. The same result can be exactly obtained by inspecting the conservation symmetry of the model [21].
For the ADS, the exact analytical solution in $d=2$ yields the exponents $\tau_{s}=4 / 3, \tau_{t}=3 / 2$ and $D=3 / 2$ [16. The upper critical dimension is found to be $d_{c}=3$, and it is also possible to find exactly the logarithmic corrections to scaling 16,22 . The introduction of stochastic ingredients in the toppling dynamics of directed sandpiles has been studied only recently in a model that randomly stores energy on each toppling [17]. This model is strictly related to directed percolation and defines a universality class "per se". In our case stochasticity affects only the partition of energy during topplings, and there is no analytical insight for the critical behavior of this model. In order to discriminate between ADS and SDS we perform simulations of both models for sizes ranging from $L=100$ to $L=6400$. Statistical distributions are obtained averaging over $10^{7}$ avalanches. Comparison of numerical results on the ADS allows us to check the reliability and degree of convergence with respect to the lattice sizes used.

It is well known from the many numerical papers on sandpiles that an accurate determination of the exponents $\tau_{s}$ and $\tau_{t}$ is a subtle issue. An overall determination within a $10 \%$ of accuracy is a relatively easy task. However, a truly accurate measurement, allowing a precise discrimination of universality classes, is strongly affected by the lower and upper cut-offs in the distribution. Extrapolations and local slope analysis are often very complicated and the relative error bars are not clearly defined. In this respect, it is far better to calculate exponents by methods that contain the system-size dependence explicitly; namely data collapse and moment analysis. Moment analysis was introduced by De Menech et al. 23] in the context of the two dimensional BTW, and it has been used extensively on Abelian and stochastic models 15,24. The $q$-moment of the avalanche size distribution on a lattice of size $L,\left\langle s^{q}\right\rangle_{L}=\int s^{q} P(s) d s$, has the following size dependence

$$
\left\langle s^{q}\right\rangle_{L}=L^{D\left(q+1-\tau_{s}\right)} \int y^{q-\tau_{s}} \mathcal{G}(y) d y \sim L^{D\left(q+1-\tau_{s}\right)},
$$

where we have used the transformation $y=s / L^{D}$ in the finite size scaling (FSS) form Eq. (11). More generally, $\left\langle s^{q}\right\rangle_{L} \sim L^{\sigma_{s}(q)}$, where the exponents $\sigma_{s}(q)$ can be obtained as the slope of the log-log plot of $\left\langle s^{q}\right\rangle_{L}$ versus $L$. Using Eq. (3), we obtain $\left\langle s^{q+1}\right\rangle_{L} /\left\langle s^{q}\right\rangle_{L} \sim L^{D}$ or $\sigma_{s}(q+1)-\sigma_{s}(q)=D$, so that the slope of $\sigma_{s}(q)$ as a function of $q$ is the cutoff exponent; i.e. $D=\partial \sigma_{s}(q) / \partial q$. This is not true for small $q$ because the integral in Eq. (3) is dominated by its lower cutoff. In particular, corrections to scaling are important for $q \leq \tau_{s}-1$. An additional and strong check on the numerical data can be found in the fact that, as we have previously shown, the first moment of the size distribution must scale linearly with $L$. This last constrain also allows the evaluation of the the exponent $\tau_{s}$ from the scaling relation $\left(2-\tau_{s}\right) D=\sigma_{s}(1)=1$, that should be satisfied for large enough sizes.

Along the same lines we can obtain the moments of the avalanche time distribution. In this case $\left\langle t^{q}\right\rangle_{L} \sim L^{\sigma_{t}(q)}$, 


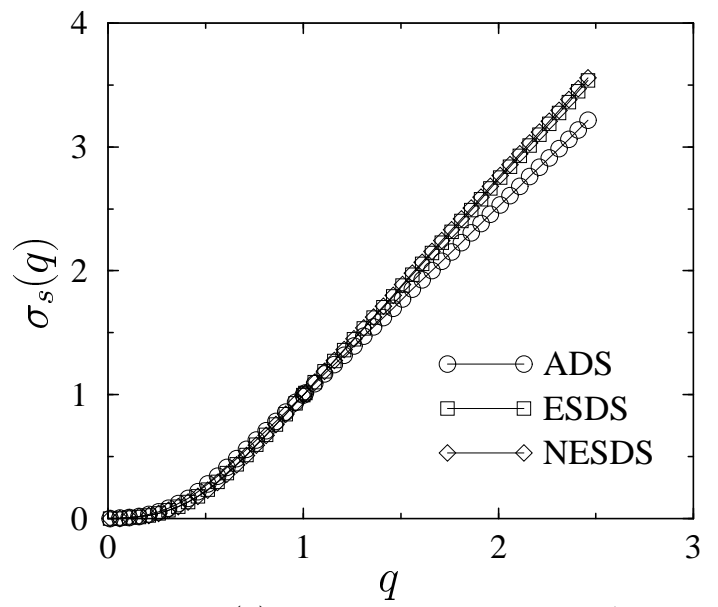

FIG. 2. Plot of $\sigma_{s}(q)$ for the $d=2$ models ADS, ESDS, and NESDS.

with $\partial \sigma_{t}(q) / \partial q=z$. Analogous considerations for small $q$ apply also for the time moment analysis. Here, an estimate of the asymptotic convergence of the numerical results is provided by the constraint $z=1$, that holds for large enough sizes. Then, the $\tau_{t}$ exponent can be found using the scaling relation $\left(2-\tau_{t}\right)=\sigma_{t}(1)$.

Despite the fact that the moment method is usually rather accurate, it must be corroborated by a data collapse analysis. The FSS of Eqs. (11,2) has to be verified and must be consistent with the numerical exponents obtained from the moment analysis. This can be done by rescaling $s \rightarrow s / L^{D}$ and $P(s) \rightarrow P(s) L^{D \tau_{s}}$ and correspondingly $t \rightarrow t / L^{z}$ and $P(t) \rightarrow P(t) L^{z \tau_{t}}$. Data for different $L$ must then collapse onto the same universal curve if the FSS hypothesis is satisfied. Complete consistency between the methods gives the best collapse with the exponents obtained by the moments analysis. In Table 1 we report the exponents found for the ADS, ESDS and NESDS in $d=2$. Figure 2 shows the moments $\sigma_{s}(q)$. Figures 3 and 14 plot the FSS data collapse for sizes and times, respectively.

The exponents obtained for the ADS are in perfect agreement with the expected analytical results. This fact supports the idea that the system sizes used in the present work allow to recover the correct asymptotic behavior. It is worth remarking that, for small and medium lattice sizes, both moments and data collapse analysis present scaling features that can not be reconciled in the single scaling picture usually considered. These anoma-

\begin{tabular}{ccccc}
\hline \hline Model & $\tau_{s}$ & $D$ & $\tau_{t}$ & $z$ \\
\hline DR & $4 / 3$ & $3 / 2$ & $3 / 2$ & 1 \\
ADS & $1.34 \pm 0.01$ & $1.51 \pm 0.01$ & $1.51 \pm 0.02$ & $1.00 \pm 0.01$ \\
ESDS & $1.43 \pm 0.01$ & $1.74 \pm 0.01$ & $1.71 \pm 0.03$ & $0.99 \pm 0.01$ \\
NESDS & $1.43 \pm 0.01$ & $1.75 \pm 0.01$ & $1.74 \pm 0.04$ & $0.99 \pm 0.01$ \\
\hline \hline
\end{tabular}

TABLE I. Critical exponents for directed sandpiles in $d=2$. DR: Dhar and Ramaswamy's exact result; ADS: Abelian model; ESDS, NEDSD: stochastic models.
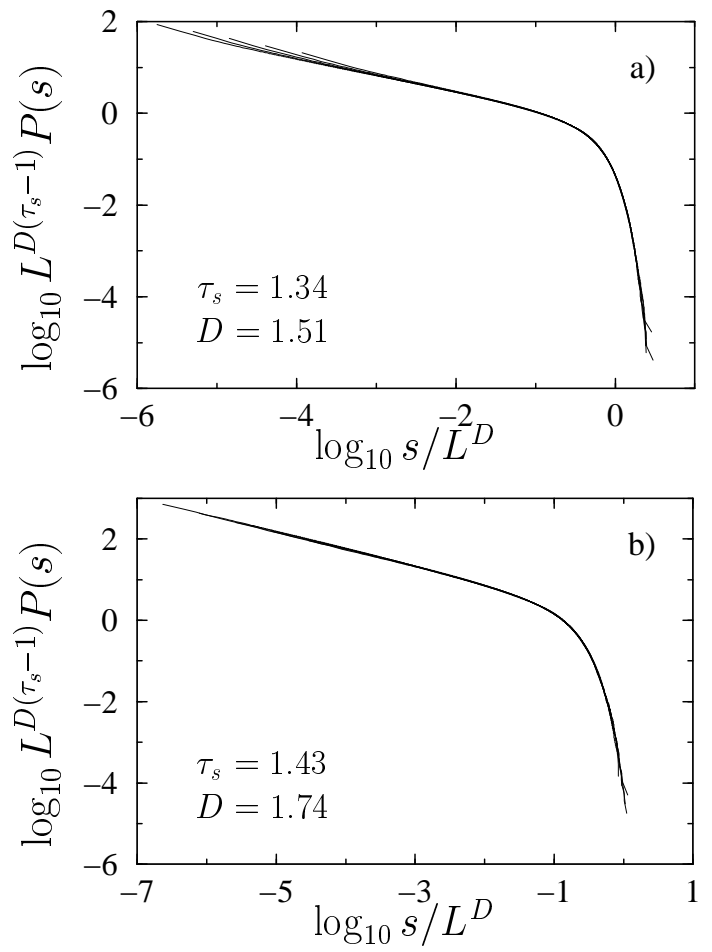

FIG. 3. Data collapse analysis of the integrated avalanche size distribution for the $d=2$ models a) ADS and b) ESDS. System sizes are $L=400,800,1600,3200$, and 6400 . The results for the ESDS and NESDS are identical, within error bars.

lies are not persistent and disappear for reasonably large sizes $\left(L \simeq 10^{3}\right)$. This evidence for a slow decaying of finite size effects could shed light into several anomalies reported in isotropic sandpiles, for which, unfortunately, it is very difficult to reach very large sizes $[15,23,24]$. Results for the ESDS and NESDS are identical within the error bars, indicating that these two models are in the same universality class. On the other hand, the obtained exponents show, beyond any doubt, that Abelian and stochastic directed sandpile models do not belong to the same universality class.

The compelling numerical evidence for two distinct universality classes does not tell us what is the basic mechanism at the origin of the different critical behavior. In order to have a deeper insight into the dynamics of the various models, we have inspected the geometric structure of the resulting avalanches. In Fig. 5 we depict in a color plot the local density of topplings in two avalanches of size 50000 corresponding to the twodimensional ADS and ESDS models. From the figure it becomes apparent that the stochastic dynamics introduces multiple toppling events, which are by definition absent in the Abelian case. This gives rise to very different avalanche structures, eventually reflected in the asymptotic critical behavior. In particular, the fractal dimension $D$ is indicative of the scaling of toppling events with sizes. In the stochastic case we recover a higher 

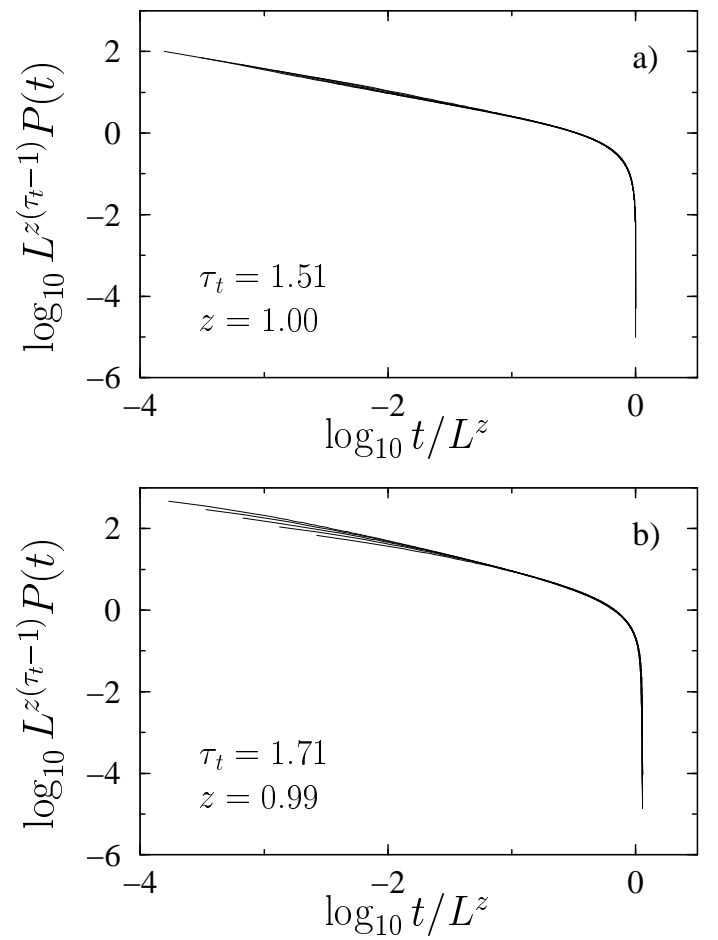

FIG. 4. Data collapse analysis of the integrated avalanche time distribution for the $d=2$ models a) ADS and b) ESDS. System sizes are $L=400,800,1600,3200$, and 6400. The results for the ESDS and NESDS are identical, within error bars.

fractal dimension than in the Abelian case. The multiple toppling mechanism has been proposed in the past as the origin of differences between isotropic Abelian and stochastic sandpiles as well. In that case, however, multiple toppling is a common feature of both models, and for the largest sizes reached so far share, they share the same fractal dimension $D$ [15].

Analysis of the models in three dimensions is strongly hindered by the presence of logarithmic corrections 116,22. Nonetheless, a naive application of the moment analysis yields values compatible with the mean-field results $\tau_{s}=3 / 2, \tau_{t}=2$, and $D=2$ [16]. More interestingly, in Ref. 16] the authors were able to deduce the exact form of the logarithmic corrections in $d=3$ for the avalanche time distribution, namely $P(t) \sim t^{-2} \ln t$. In Figure 6 we have checked that the same logarithmic corrections apply to both the Abelian and ESDS sandpiles. This remarkable fact lends support to the critical dimension of the stochastic model being $d_{c}=3$.

In summary, we have reported large scale numerical simulations of a stochastic directed sandpile model. This model defines unambigously a different universality class with respect to the Abelian directed sandpile model. The origin of this difference is traced back to the avalanche cluster geometric structure, providing new clues to understand the effect of stochastic elements in the dynamics

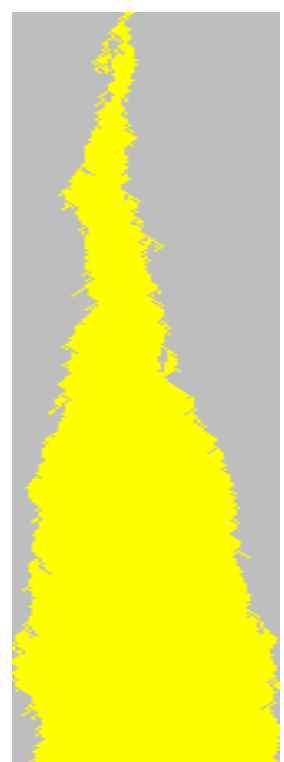

a)

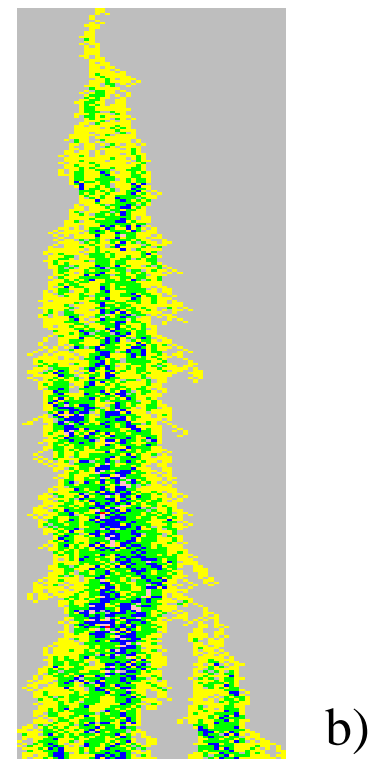

FIG. 5. Color plots of the local density of topplings in two avalanches of size 50000 , in $d=2$, for a) ADS and b) ESDS. Yellow represents a single toppling per site; red stands for the maximum number of topplings.

of avalanche processes.

This work has been supported by the European Network under Contract No. ERBFMRXCT980183. We thank D. Dhar, D. Dickman, M. A. Muñoz, A. Stella, and S. Zapperi for helpful comments and discussions.

[1] P. Bak, C. Tang, and K. Wiesenfeld, Phys. Rev. Lett. 59, 381 (1987); Phys. Rev. A 38, 325 (1988).

[2] H. J. Jenssen, Self-Organized Criticality (Cambridge University Press, Cambridge, 1998).

[3] K. B. Lauritsen, S. Zapperi, and H. E. Stanley, Phys. Rev. E 54, 2483 (1996).

[4] A. Vespignani and S. Zapperi, Phys. Rev. E 57, 6345 (1998).

[5] M. Paczuski and S. Boettcher, Phys. Rev. Lett. 77, 111 (1996).

[6] K. B. Lauritsen and M. Alava, cond-mat/9903346.

[7] R. Dickman, A. Vespignani, and S. Zapperi, Phys. Rev. E 57, 5095 (1998); A. Vespignani, R. Dickman, M. A. Muñoz, and S. Zapperi, Phys. Rev. Lett. 81, 5676 (1998).

[8] This connection has been also discussed for the BakSneppen SOC model. S. Maslov, M. Paczuski, and P. Bak, Europhys. Lett. 27, 97 (1994); P. Grassberger, Phys. Lett. A 200, 277 (1995).

[9] S. S. Manna, J. Phys. A 24, L363 (1991).

[10] A. Díaz-Guilera, Phys. Rev. A 45, 8551 (1992).

[11] A. Vespignani, S. Zapperi, and L. Pietronero, Phys. Rev. E 51, 1711 (1995). 

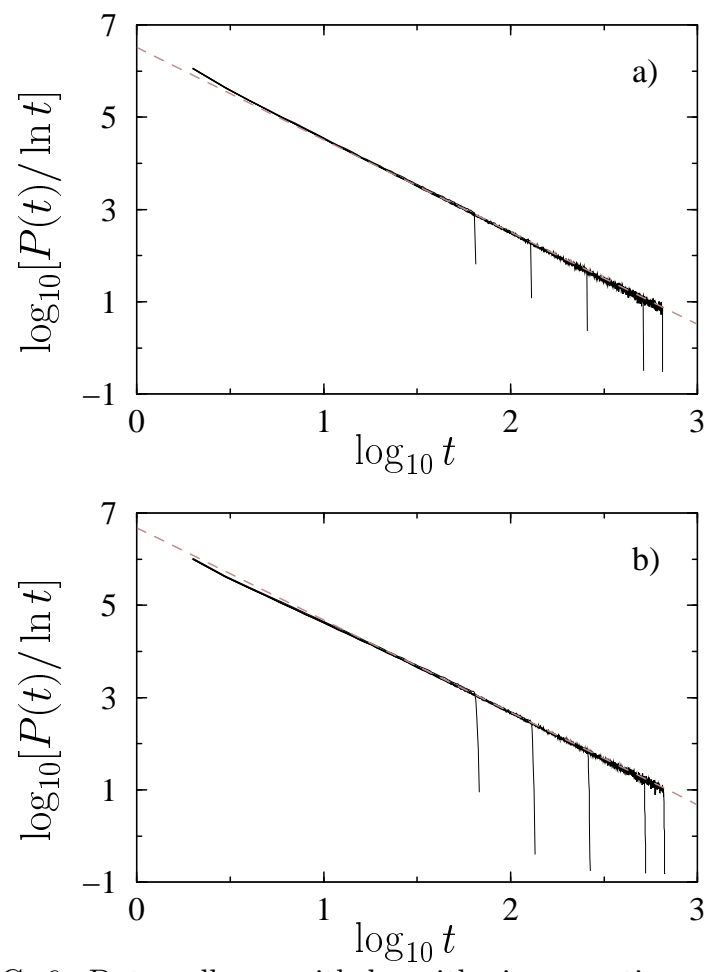

FIG. 6. Data collapse with logarithmic corrections of the avalanche time distribution for the $d=3$ models a) ADS and b) ESDS. The dashed lines have slope -2 . System sizes are $L=64,128,256,512$, and 650 .

[12] J. Hasty and K. Wiesenfeld, Phys. Rev. Lett. 81, 1722 (1998).

[13] P. Grassberger and S. S. Manna, J. Phys. (France) 51, 1077 (1990).

[14] E. Milshtein, O. Biham, and S. Solomon, Phys. Rev. E 58, 303 (1998).

[15] A. Chessa, H. E. Stanley, A. Vespignani, and S. Zapperi, Phys. Rev. E 59, R12 (1999).

[16] D. Dhar and R. Ramaswamy, Phys. Rev. Lett. 63, 1659 (1989).

[17] B. Tadić and D. Dhar, Phys. Rev. Lett. 79, 1519 (1997).

[18] D. Dhar, Physica A 263, 4 (1999).

[19] L. P. Kadanoff, S. R. Nagel, L. Wu, and S. Zhou, Phys. Rev. A 39, 6524 (1989).

[20] T. Tsuchiya and M. Katori, J. Phys. A: Math. Gen. 32, 1629 (1999).

[21] R. Pastor-Satorras and A. Vespignani, in preparation.

[22] S. Lübeck, Phys. Rev. E 58, 2957 (1998).

[23] M. De Menech, A. L. Stella, and C. Tebaldi, Phys. Rev. E 58, R2677 (1998); C. Tebaldi, M. De Menech, and A. L. Stella, cond-mat/9903270.

[24] A. Chessa, E. Marinari, A. Vespignani, and S. Zapperi, Phys. Rev. E 57, R6241 (1998); A. Chessa, A. Vespignani, and S. Zapperi, cond-mat/9811365. 